\title{
Use of optical tweezers technology for long-term, focal stimulation of specific subcellular neuronal compartments $\dagger$
}

\author{
Elisa D'Este, $\ddagger^{a}$ Gabriele Baj, ${ }^{b}$ Paolo Beuzer, $\S^{a}$ Enrico Ferrari, $\uparrow^{a}$ Giulietta Pinato, ${ }^{a}$ \\ Enrico Tongiorgi ${ }^{b}$ and Dan Cojoc ${ }^{* a}$
}

Received 12th September 2010, Accepted 21st February 2011

DOI: $10.1039 / \mathbf{c 0 i b 0 0 1 0 2 c}$

Spatial regulation of secretory molecule release is a sophisticated mechanism used by the nervous system to control network development and finely tune the activity of each synapse. Great efforts have been made to develop techniques that mimic secretory molecule release with the aim of stimulating neurons as close as possible to physiological conditions. However, current techniques have poor spatial resolution or low flexibility. Here, we propose a novel approach to achieve focal and prolonged stimulation of neurons using optical tweezers and single microbeads functionalized with a secretory molecule, the neurotrophin brain-derived neurotrophic factor (BDNF). BDNF is a key regulator of neuronal development and plasticity. We show that single BDNF-coated microbeads can be extracted with optical tweezers from small reservoirs and positioned with submicrometric precision to specific sites on the dendrites of cultured hippocampal neurons. Localized contact of microbeads functionalized with BDNF, but not with bovine serum albumin (BSA), induced focal increase of calcium signaling in the stimulated dendrite, specific activation of the TrkB receptor pathway and influenced the development of growth cones. Remarkably, a single BDNF-coated bead localized on a dendrite was found to be enough for TrkB phosphorylation, an efficient and long-lasting activation of calcium signaling in the soma, and c-Fos signaling in the nucleus, comparable to bath stimulation conditions. These findings support the use of optical tweezer technology for long-term, localized stimulation of specific subcellular neuronal compartments.

${ }^{a}$ Istituto Officina dei Materiali IOM-CNR, Laboratorio TASC, Area Science Park-Basovizza, S.S. 14 km 163.5, 34149 Trieste, Italy.E-mail:cojoc@tasc.infm.it

${ }^{b}$ BRAIN Centre for Neuroscience, Department of Life Sciences, University of Trieste, via Giorgieri 5, 34127 Trieste, Italy

$\dagger$ Electronic supplementary information (ESI) available. See DOI: $10.1039 / \mathrm{c} 0 \mathrm{ib} 00102 \mathrm{c}$

$\ddagger$ Also with: Dipartimento di Fisica, University of Trieste, Italy.

$\S$ Present address: Institut Curie, 26 rue d'Ulm, Paris, France.

- Present address: MRC Laboratory of Molecular Biology, Hills Road, Cambridge, UK.

\section{Introduction}

Neurons are highly polarized cells with well defined and specialized compartments, each having specific receptors and functions. Stimulation through bath administration of molecules, therefore, does not reflect physiological conditions in which molecules are released at low concentrations and in a spatially restricted fashion. In this context, developing new methods to study focal stimulation, with both control and experiment on the same cell, represents a fundamental goal in neurobiology.

\section{Insight, innovation, integration}

To study the neuronal response of a single cell to a locally applied biochemical stimulus, we developed an optical tweezers procedure to trap and position functionalized micrometric vectors with high spatial resolution on the cell. By settling several small reservoirs in the observation chamber, single differently functionalized vectors could be selected to compare their different effects. We demonstrated that even a single vector is able to induce a cellular activation. Long-term stimulation and monitoring of the cells revealed positive responses both in the soma and the dendrites. These achievements allow the design of experiments in which local stimulation at spine level is possible, with a non-expensive setup that can be easily implemented in any bio-laboratory. 
While for ions, neurotransmitters and other small molecules, focal release can be achieved by photo-uncaging, ${ }^{1}$ this has been proved more difficult for proteins, even if new proposed techniques involving modified photo-activatable amino acids ${ }^{2}$ or entire protein domains are applied. ${ }^{3}$ If uncaging is carried out in the culture medium, released molecules may reach neighboring synapses on the same cell or even on other cells in close proximity. Uncontrolled spillover is a significant drawback of the available delivery techniques, especially for secretory molecules that can affect both pre- and post-synaptic cells like neurotrophic factors. One of these factors is brain-derived neurotrophic factor (BDNF), a member of the neurotrophins family that promotes either survival, growth and differentiation or cell death, depending on whether it occurs as the precursor, which activates the receptor p75NTR, or as the mature form, which binds preferentially to TrkB receptors. ${ }^{4}$ The cellular response to BDNF depends also on the subcellular localization of p75NTR and TrkB receptors, since they are differentially expressed on the surface of the cell. Moreover, they can be inserted at the cell surface or internalized depending on the neuronal activity and can trigger different intracellular pathways according to a specific subcellular compartment or biochemical environment. ${ }^{5-7}$

Attempts to achieve local control of neurotrophin concentration include the use of a caged antibody against BDNF, through which a decrease of potentiation for several synapses has been obtained in the range of hundreds of microns from the uncaging point. ${ }^{8}$ Alternative methods are the use of neurotrophin donor-recipient pairs of neurons, ${ }^{9}$ micropipettes and Picospritzer delivery ${ }^{10}$ or coated beads. ${ }^{11-14}$ For instance, large beads $(10 \mu \mathrm{m})$ cross-linked with BDNF and manipulated with micropipettes have been used to potentiate the nearby synapses. ${ }^{12,14}$ These techniques, however, provide minimal flexibility and low spatial resolution.

Optical tweezers (OT) have been proved as a valuable tool in biology, ${ }^{15}$ being widely employed as a tool to confine or constrain single cells, as well as to organize, assemble, locate, sort and modify them. ${ }^{16}$ Optically trapped microbeads have been used to mechanically stress cell membranes, e.g. erythrocytes, ${ }^{17} \mathrm{HeLa}$ cell membranes, ${ }^{18}$ and as probes to measure $\mathrm{pN}$ forces exerted by filopodia of $\mathrm{J} 774$ macrophages ${ }^{19}$ or filopodia and lamellipodia of the neuronal growth cones. ${ }^{20}$ Chemical stimuli, encapsulated in optically manipulated liposomes and delivered by breaking the liposome with a pulsed UV laser have been proposed for fast and local delivery. ${ }^{21}$ Recently, optically manipulated microsources to generate spatial and time adaptable molecular gradients have been applied to control neutrophil migration. ${ }^{22}$

OT are a flexible tool, in the sense that particles from different materials and in a wide size range $(20 \mathrm{~nm}-20 \mu \mathrm{m})$ can be trapped and manipulated. ${ }^{23}$ OT can also be easily coupled with other imaging techniques in optical microscopy, e.g. fluorescence imaging. Since a high numerical aperture (NA) lens is used in optical trapping, the spatial resolution for particle manipulation is about half a micron which, for cells with sizes of tens of microns, can be considered as highresolution. Here, based on the flexibility and high-resolution offered by optical tweezers, we propose a novel approach to apply local stimulation at a precise location on a neuron.
In particular, we show that two single silica bead vectors coated with either BDNF or bovine serum albumin (BSA) could be trapped, pulled out from two different reservoirs and placed on two different dendrites from the same neuron obtaining a significant increase in calcium levels within the BDNF-stimulated, but not the control, dendrite.

\section{Materials and methods}

\section{Cell cultures}

Primary hippocampal neurons were prepared from $\mathrm{P} 0-\mathrm{P} 1$ rats as described previously ${ }^{24}$ with slight modifications. Cells were plated on poly-ornithine and 2\% Matrigel (BD Biosciences) coated homemade glass bottom Petri dishes at a density of $10^{5}$ cells $\mathrm{mL}^{-1}$ per dish. Neurons were cultured for at least 5 days in a $5 \% \mathrm{CO}_{2}$-humidified incubator, in Minimum Essential Medium with Earle's salts and Glutamax I (MEM, Life Technologies, Invitrogen) with 10\% FBS (Gibco), $35 \mathrm{mM}$ D-glucose (Lancaster), $14 \mathrm{mM}$ Hepes (Sigma), $1 \mathrm{mM}$ vitamin B12 (Sigma), $0.36 \mathrm{mM}$ d-Biotin (Sigma), $30 \mu \mathrm{g} \mathrm{mL}^{-1}$ insulin (Sigma), $100 \mu \mathrm{g} \mathrm{mL}^{-1}$ bovine transferrin (Sigma), and antibiotics (Euroclone). The medium was changed every 2 days from the second day in culture onwards.

\section{Bead functionalization}

$1.5 \mu \mathrm{m}$ large silica beads coated with $\mathrm{COOH}$ groups (Kisker-biotech, cat PSi-1.5COOH) were functionalized using PolyLink Protein Coupling Kit (Bangs Laboratories Inc., cat PL01N) following the manufacturer's protocol. Briefly, about $1.4 \times 10^{5}$ beads were incubated with $500 \mathrm{ng}$ of BSA or BDNF in the presence of $20 \mu \mathrm{g} \mu \mathrm{L}^{-1}$ EDAC for $1 \mathrm{~h}$ at room temperature and stored in storage buffer at $4{ }^{\circ} \mathrm{C}$.

\section{ELISA}

The ELISA test was performed using BDNF $\mathrm{E}_{\max }{ }^{{ }^{R}}$ ImmunoAssay Systems (Promega, cat G7611), following the manufacturer's protocol. Supernatants, obtained after bead functionalization, were diluted $1: 10$ and three washes were analyzed. Release of BDNF was also tested after the beads were treated in different conditions, i.e. normal storage at $4{ }^{\circ} \mathrm{C}$, $1 \mathrm{~h}$ at $37^{\circ} \mathrm{C}$ or $5 \mathrm{~min}$ at $100^{\circ} \mathrm{C}$. This latter condition was also used to test whether the immunoassay was able to detect the protein after it was boiled.

\section{Immunohistochemistry}

Coated microspheres were washed in PBS and incubated for $1 \mathrm{~h}$ at room temperature with anti-BDNF or anti-BSA (both $1: 50$, Sigma). Beads were then centrifuged, washed and incubated for $30 \mathrm{~min}$ at room temperature with anti-rabbit Alexa 488 (Invitrogen) and finally analyzed using confocal microscopy (Nikon $\mathrm{C} 1-\mathrm{Si}$ ).

For pTrkB and c-Fos analysis, neurons were incubated either with functionalized beads, BDNF $50 \mathrm{ng} \mathrm{mL} \mathrm{m}^{-1}$ or K252a $200 \mathrm{nM}$ for $15 \mathrm{~min}$. Neurons were then washed in PBS, fixed in PFA 4\% for 15 min, permeabilized with Triton $\mathrm{X}-1000.1 \%$ for $15 \mathrm{~min}$ and then incubated with primary antibodies (anti-c-Fos antibody $1: 50$, Lab Vision; anti-BDNF $1: 50$, Sigma; anti-BSA 1:50, Sigma; anti-pTrkB $1: 250$, kind 
a
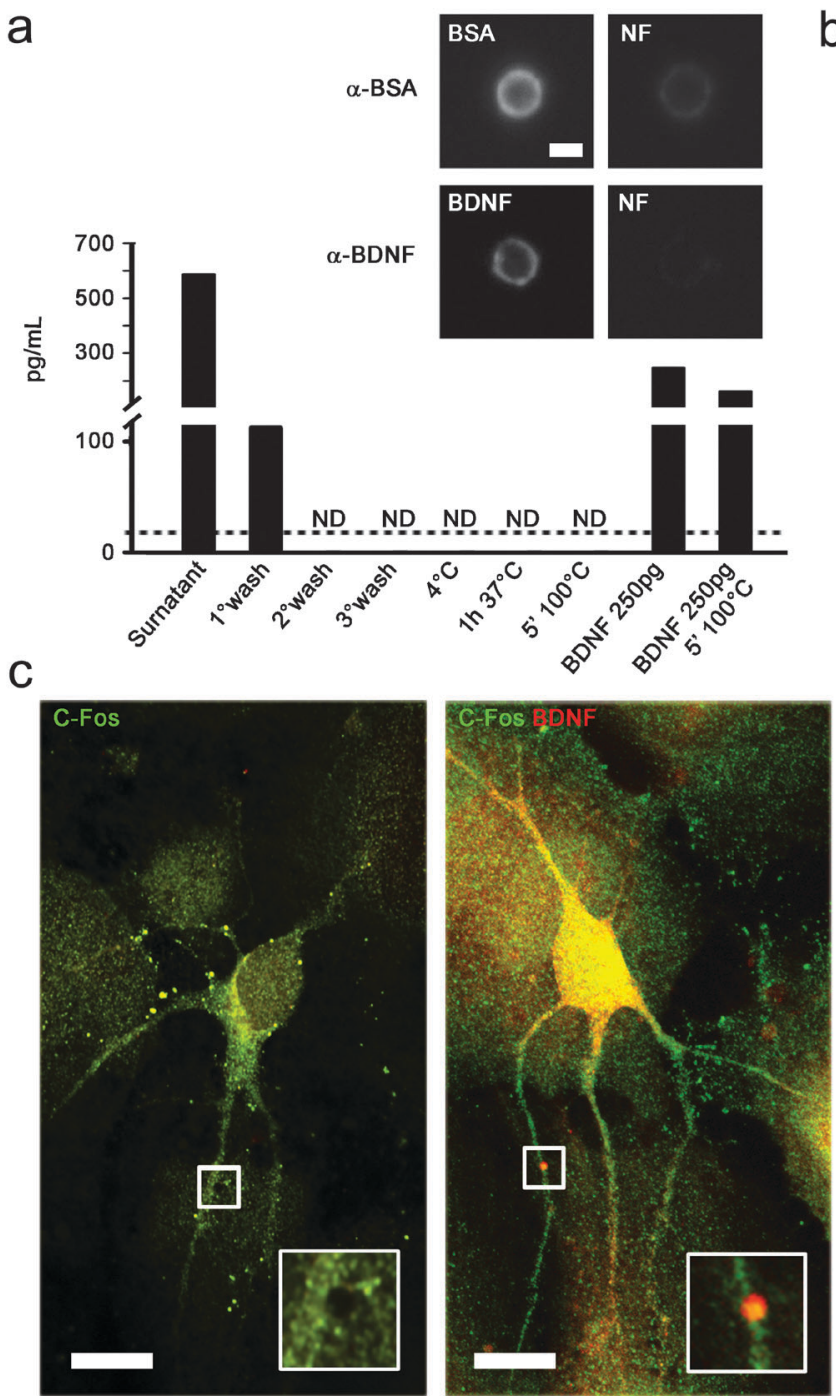

b

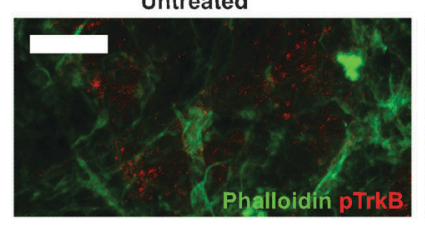

BSA beads
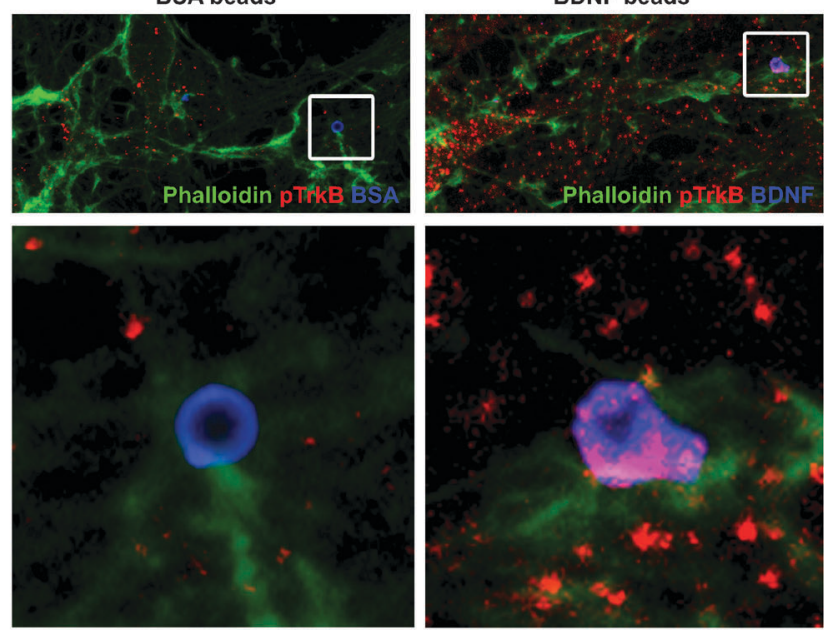

d

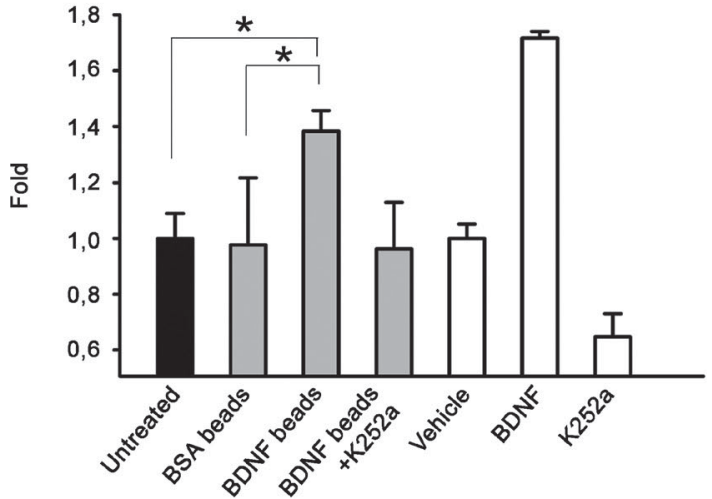

Fig. 1 BDNF-coated beads trigger TrkB signaling pathway. (a) ELISA assay on the supernatants (1:10 dilution) of beads collected right after the functionalization, the washes or the indicated conditions. Dashed line indicates the threshold of sensitivity on the test (ND - not detectable). Immunofluorescence images of BSA- and BDNF-beads (left) and of non-functionalized beads (NF) (right) incubated with the corresponding primary antibodies are shown at the upper-right corner. Scale bar $1 \mu \mathrm{m}$. (b) Confocal microscopy images showing that no activation of the TrkB (pTrkB) is detectable for the control (untreated neurons) or BSA-coated beads (images on the left, scale bar $15 \mu \mathrm{m}$ ) while TrkB is phosphorylated (red dots) for the neurons incubated with BDNF $50 \mathrm{ng} \mathrm{mL}^{-1}$ or BDNF-beads (images on the right). Magnified images at the bottom show the difference between the pTrkB induced by BSA and BDNF coated beads. The actin is marked by phalloidin (green) while the BSA and BDNF are in blue. (c) Neurons touched by BSA (left) and BDNF (right) beads have negative and positive, respectively, nuclear staining for c-Fos. Enlarged images of the region surrounding the beads are shown in insets. Rat hippocampal neurons $\mathrm{P} 0-1,6$ days in vitro, prepared as described in methods have been used. Scale bars $10 \mu \mathrm{m}$. (d) c-Fos positive neurons for different treatments were counted. Untreated cells, mean of the counts per experiment, $n_{\text {mean }}=705$. BSA-beads (control) $n_{\text {mean }}=91$, BDNF-beads $n_{\text {mean }}=94$, BDNF-beads + K $252 \mathrm{a} n_{\text {mean }}=68$. The last three bars refer to bath experiments: vehicle $n_{\text {mean }}=414$, BDNF $50 \mathrm{ng} \mathrm{mL}^{-1} n_{\text {mean }}=463$, K252a $200 \mathrm{nM} n_{\text {mean }}=779 . p$-value: $*<0.05$. Analysis performed with one-way ANOVA.

gift of M. V. Chao) in PBS in the presence of $2 \%$ NGS for $2 \mathrm{~h}$ at room temperature. After washing them in PBS, they were incubated for $1 \mathrm{~h}$ at room temperature with antirabbit Alexa (488 or 568) or anti-mouse Alexa (568 or 647) (Invitrogen), 5 min with Hoechst and mounted in Mowiol. In the pTrkB experiments, actin was marked using Phalloidin FITC (Sigma P8252) for $15 \mathrm{~min}$. For data analysis of the c-Fos experiments, the neurons touched by the beads and the un-touched neurons (untreated) were classified as c-Fos positive and c-Fos negative, according to the nuclear staining.
The percentage of c-Fos positive untreated neurons was normalized to 1 .

Optical manipulation. A custom optical tweezers setup was built on a mini breadboard MS12B (Thorlabs, USA) and coupled to the lateral port of an inverted microscope (Nikon TE-300), as shown in Fig. 3. The laser trapping beam (1064 nm) from an Ytterbium fiber laser YLM-5 (IPG Photonics, USA) was focused by a convergent lens $(\mathrm{L} 1, f=200 \mathrm{~mm}$ ) into the lateral port (LP) plane of the 
microscope. The trapping beam was then collimated by the microscope tube lens (TL) and focused by the microscope objective $(60 \times, 1.4 \mathrm{NA}$, Nikon) onto the sample. The sample imaging before and during trapping was accomplished on an external camera (WAT1000, Watec LTD, Japan) using a relay lens (L2, $f=60 \mathrm{~mm})$. The vectors were loaded in square capillaries with $50 \mu \mathrm{m}$ inner size (VitroCom, US, cat. 8505), which were used as reservoirs. They were pre-treated with $1 \%$ BSA to reduce beads binding to the walls.

Growth cone mobility. The experiments on growth cones were performed on 1 DIV (day in vitro) hippocampal neurons using a $100 \times 1.4$ NA oil immersion objective (Nikon) using DIC (differential interference contrast) imaging. Images were collected for $5 \mathrm{~min}$ every $20 \mathrm{~s}$ before the stimulation and for $40 \mathrm{~min}$ at the same frame rate after stimulation.

Calcium imaging. Fluorescence and transmission images were recorded by a second cooled video camera (CCD-2) (Sensicam, PCO Imaging, Germany) located at the bottom port of the microscope. The fluorescent dye used was Fluo-3, AM Ester (Biotium, Inc, USA). Neurons were incubated for 40 min with $7 \mathrm{mM}$ Fluo-3 prior to use. Images were acquired every $2 \mathrm{~min}$ with the Sensicam camera. Analysis was performed using NIH Software ImageJ (http://rsbweb. nih.gov/ij/index.html). Every stack was normalized by its background and then all values were normalized to the first stack after bead placement. For the analysis of dendrites, measurements were taken on segments of $20 \mu \mathrm{m}$ length, centered on the bead position.

\section{Results}

\section{Biological activity of functionalized beads}

Microspheres are frequently used in biology and beads of different materials, and different sizes and functionalizations are commercially available. For our experiments we chose silica beads coated with carboxylic groups, because they can be cross-linked with a variety of proteins and have a refractive index (1.45 against 1.33 of water) that makes them suitable for optical trapping. The dimension selected was $1.5 \mu \mathrm{m}$ diameter, that is similar to the width of dendrites and can be easily visualized in transmission microscopy. We cross-linked either BDNF or BSA as a control to the spheres. The carboxylic groups present on the surface of the beads formed an amide bond with the amine of the proteins in the presence of the coupling reagent EDAC (1-ethyl-3-(3-dimethylaminopropyl) carbodiimide). The reaction was performed in a large excess of protein in solution and thus we can assume that the coating has a high density of proteins. In this way the effect observed is not dependent of the number of ligands but of the number of receptors present on the cell surface. Coating of the bead surface was tested by immunofluorescence with anti-BDNF or anti-BSA antibodies (Fig. 1a). Neither primary nor secondary antibodies attached on untreated beads, confirming that the signal observed on the functionalized beads was specific. Since the amide bond between BDNF and silica is very strong, the only possible source of BDNF leakage is due to molecules adsorbed but not covalently linked to the bead surface. To rule out this possibility of leakage from the beads, we also performed an ELISA assay. Free BDNF was found at the end of the functionalization process and in the first wash but was absent in the following washes. BDNF-functionalized vectors were also stored at $4{ }^{\circ} \mathrm{C}$ for a week or kept for $1 \mathrm{~h}$ at $37{ }^{\circ} \mathrm{C}$ to mimic the conditions that will be used in the experiments. The protein was undetectable in the supernatants of these samples, showing the absence of protein leakage from the beads (Fig. 1a). Since BDNF occurs as a dimer, we finally tried to separate the monomers by boiling beads for $5 \mathrm{~min}$. In these conditions the dimers in which only one molecule is covalently bound to the bead should separate and thus be detected. We again did not detect any signal. We can thus state that BDNF never detaches from the surface of microspheres during the in vitro experiments.

In 1986, Rosenberg et al. reported the loss of biological activity of neurotrophins if their surface lysines residues were modified. ${ }^{25}$ More recent works, however, used COOH-beads functionalized with BDNF or nerve growth factor (NGF) and reported a biological effect. ${ }^{11,12,14}$ Hence, we used the same reaction to couple $\mathrm{BDNF}$ on the $\mathrm{COOH}$-beads and we tested whether after immobilization, BDNF maintained its biological function, i.e. it was able to activate TrkB receptors. TrkB activation was first demonstrated by incubating neurons with beads and visualizing TrkB phosphorylation by immunofluorescence (Fig. 1b). pTrkB dots were barely visualized in control cultures (untreated or incubated with BSA-beads) while a strong activation of the receptor was observed for the stimulated cells with BDNF (bath applied or bound to the microspheres). A particularly striking finding was the observation of spatially restricted activation of $\mathrm{pTrkB}$ on the dendrites right under the beads (Fig. 1b) which supports the effectiveness of this methodology in inducing a local stimulation of BDNF signaling. Since in our system we assume no neurotrophin internalization, and TrkB phosphorylation alone is not sufficient to demonstrate the activation of the intracellular signaling pathway, ${ }^{26}$ we analyzed the levels of c-Fos within the nucleus by immunofluorescence ${ }^{27}$ (Fig. 1c). Neurotrophins are known to induce the immediate-early gene c-Fos by pathways that do not require new protein synthesis. ${ }^{28}$ The amount of cells not in contact with beads (untreated) was normalized to 1 . Neurons contacted by BSA-beads showed nuclear levels of c-Fos similar to untreated neurons $(0.98 \pm 0.23$ s.e. $)$, while BDNF-beads induced a marked increase in nuclear c-Fos with respect to untreated neurons $(1.38 \pm 0.07 ; p$-value: 0.008$)$ and with respect to BSA-beads ( $p$-value: 0.019), similar to the effect induced by bath-applied BDNF (1.72 \pm 0.02$)$ (Fig. 1d). Incubation of neurons with the Trk inhibitor K252a abolished the effect observed $(0.96 \pm 0.17)$, indicating a specific TrkB involvement. These results demonstrated the possibility of using BDNF-coated beads as microvectors for local stimulation of neurons, since even a single bead was able to activate BDNF receptors and induce a cellular response.

Finally, we analyzed the effect of our vectors on growth cone development since BDNF is a key mediator of axonal guidance (for a review see ref. 29). Bead manipulation was achieved as explained in the next paragraph (single microbead manipulation). While BSA-beads did not influence the growth 


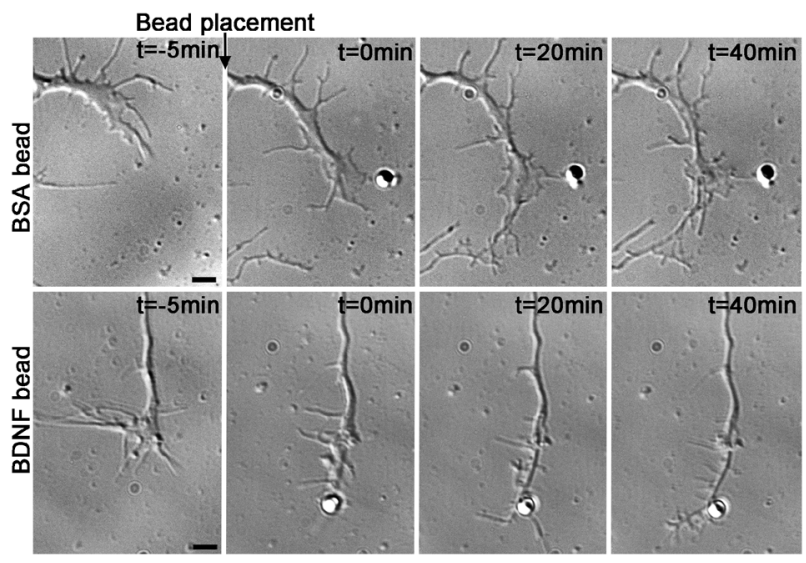

Fig. 2 Growth cone motility DIC images showing the different effects induced by BSA- and BDNF-coated beads. (Upper panel) A BSA coated bead positioned by optical manipulation on the filopodia does not affect the motility of the growth cone. (Lower panel) The BDNF coated bead stimulates the neurite development. Scale bar $2 \mu \mathrm{m}$. The first image for each case shows the neuron $5 \mathrm{~min}$ before the bead placement. Full sequences of the growth cone migrations are shown in Supplementary Movie 1 and $2 \dagger$ ). of these structures (Fig. 2 and Supplementary Movie $1 \dagger$ ), BDNF-beads were able to affect the development of the growth cones guiding the migration of lamellipodia and filopodia (Fig. 2 and Supplementary Movie $2 \dagger$ ).

\section{Single microbead manipulation}

To bring a single functionalized bead to the desired site on a neuron, we built a custom optical tweezers setup that can be easily coupled to the lateral port of an inverted microscope (Fig. 3). The laser beam at $1064 \mathrm{~nm}$ wavelength was focused by a lens and reflected by a mirror and a dichroic mirror into the lateral port of the microscope. The tube lens of the microscope collimated the beam which was then focused on the sample by the objective $60 \times$ (NA 1.4). During trapping, the sample was imaged using a first CCD camera (CCD-1). After bead positioning on the cell, achieved by optical manipulation, the mirror inside the microscope was switched vertically to allow fluorescence and transmission imaging through the direct port below the microscope, on a second more sensitive camera (CCD-2).

Since the optical trap remained fixed, its position relative to the sample cell could be changed by moving the stage of the microscope (Fig. 3 insert). BSA- and BDNF-coated beads

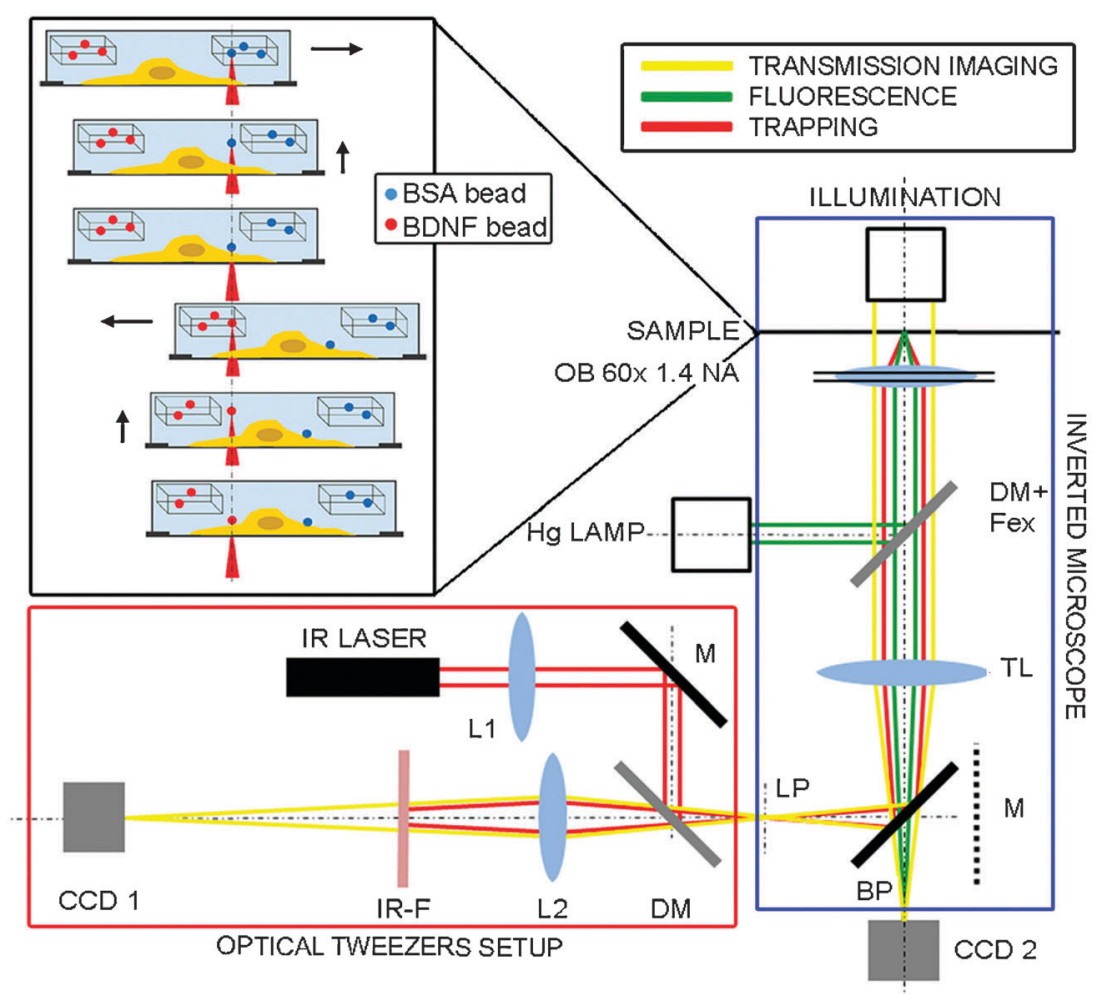

Fig. 3 Optical manipulation and calcium imaging experimental setup. A custom optical tweezers setup (red box) is coupled to the lateral port (LP) of the inverted microscope Nikon Eclipse TE300 (blue box). The colors indicate the different optical paths: trapping beam in red, transmission in yellow, fluorescence in green. During trapping the sample is imaged on the CCD-1 camera, using the relay optics (L2). Calcium imaging is recorded on the CCD-2 camera, turning out the microscope mirror (M). L1, L2: lenses; M: mirror; DM: dichroic mirror; TL: tube lens; OB: objective $(60 \times$ PlanApo NA 1.4); CCD: charge coupled device; IR-F: infrared filter; Fex: excitation filter. Inset: Layout of bead manipulation. Two reservoirs, one with BSA beads (blue) and the other with BDNF beads (red) are laid on the bottom of the cell chamber. A BSA-bead is trapped inside the reservoir and then pulled out and positioned at the desired site of the cell by moving the stage of the microscope. The same sequence is repeated to trap and position the BDNF-bead from the second reservoir. 
were prepared and stored separately in small reservoirs (square capillaries, $50 \mu \mathrm{m}$ inner size). The capillaries were laid on the bottom of the cell culture dish. Single beads were optically trapped inside the reservoirs, pulled out and then positioned with sub-micrometric resolution to the desired sites on the neurons selected for stimulation (Fig. 3 insert and Supplementary Movie 3†).

\section{Effect of bath applied BDNF}

TrkB is known to activate PLC $\gamma 1$ which produces IP3, leading to $\mathrm{Ca}^{++}$release from internal stores. ${ }^{6}$ Hence, to obtain the experimental evidence that stimulation of the BDNF/TrkB pathway after bead manipulation with optical tweezers had occurred, we measured calcium levels in the neuronal soma and dendrites. To make sure that stimulation with BDNF could produce measurable calcium signals in our experimental system, we first carried out an experiment with bath application of $50 \mathrm{ng} \mathrm{mL} \mathrm{m}^{-1}$ BDNF. Basal fluorescence of neurons loaded with Fluo-3 dye was monitored for $10 \mathrm{~min}$, acquiring images every $2 \mathrm{~min}$. BDNF was then bath-applied and fluorescence was recorded for $40 \mathrm{~min}$. A rapid rise in the calcium signal both in the soma and dendrites was observed after the stimulation (Fig. 4a). A plot of the normalized fluorescence values showed this progressive increase in the calcium signal that reached a plateau at about 15 min after BDNF stimulation in both the soma and dendrites (Fig. 4b).

\section{Dendrite stimulation with sub-micrometric resolution}

For focal stimulation experiments, we first placed a BSA-bead on a dendrite and recorded basal Fluo-3 fluorescence for $10 \mathrm{~min}$, acquiring images every $2 \mathrm{~min}$. A BDNF-coated bead was then positioned on another dendrite of the same neuron, at the same distance from the soma, and fluorescence images were acquired every $2 \mathrm{~min}$ for a period of $40 \mathrm{~min}$, as done for bath application. An example of the stimulation of $\mathrm{Ca}^{++}$ signaling induced in a pyramidal neuron is illustrated in Fig. 5. To assure that contact between the beads and dendrites had occurred, transmission images in phase contrast were acquired immediately after positioning the beads (Fig. 5, $t=-10^{\prime}$ and $\left.t=0^{\prime}\right)$. Only a low $\mathrm{Ca}^{++}$signal fluctuation was observed after BSA-bead positioning $\left(t=-9^{\prime} ; t=-5^{\prime} ; t=-1^{\prime}\right)$. On the contrary, the fluorescence signal clearly increased in the cell body after positioning the BDNF-bead. Moreover, a difference in the signal obtained from the two stimulated dendrites was recognized, indicating a selective activation of the BDNF-stimulated neurite (Fig. 5, inset).

To rule out the possibility that the effect observed with BDNF-beads could be due to the mechanical stimulation of the dendrites, we ran a set of control experiments in which a pair of BSA-beads were positioned on two different dendrites of the same neuron. An example of time lapse analysis of calcium fluorescence is illustrated in Supplementary Fig. $1 \dagger$,
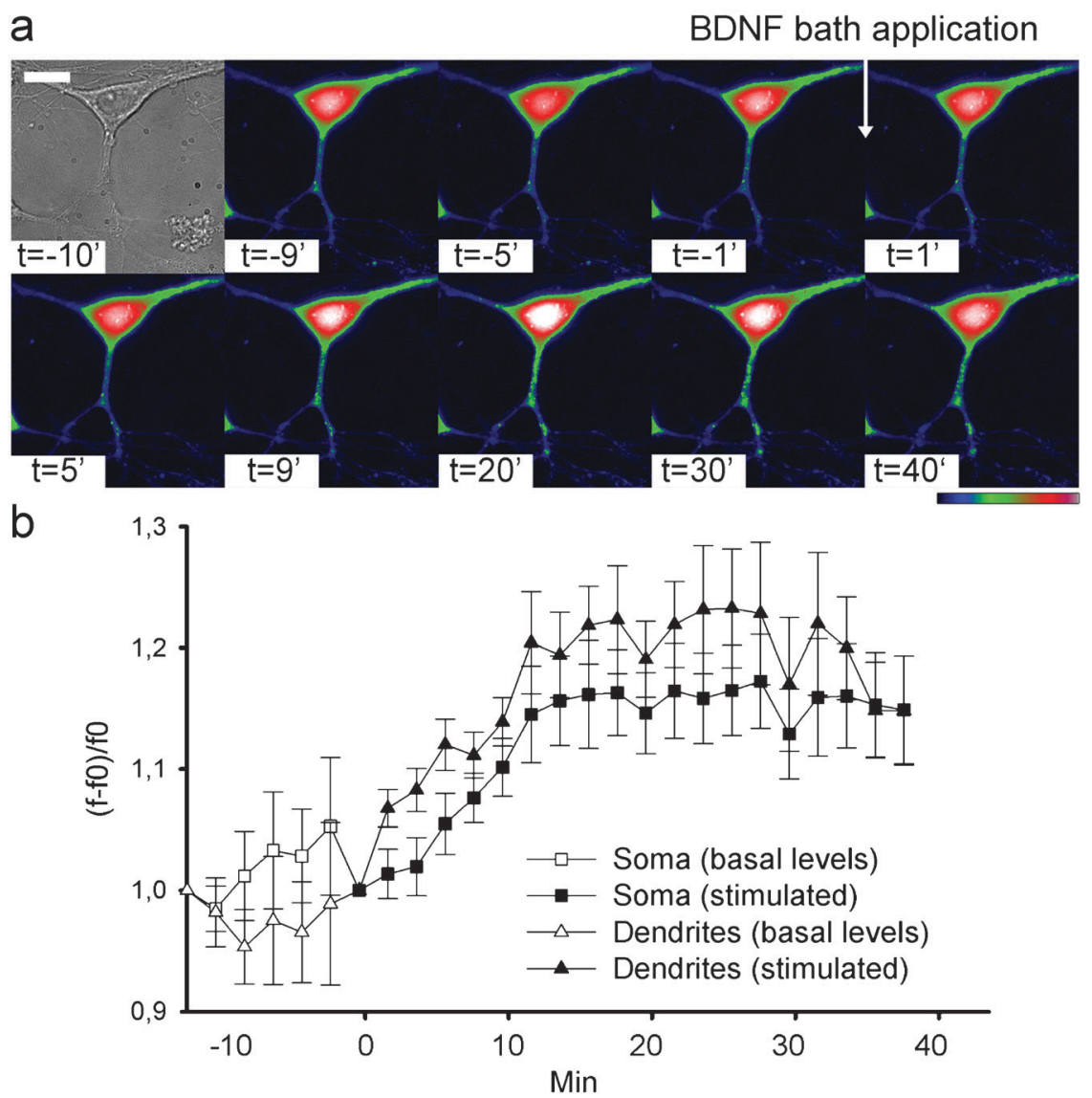

Fig. 4 The effect of BDNF bath administration. $50 \mathrm{ng} \mathrm{mL}^{-1}$ BDNF induced an increase in calcium levels. (a) Transmission image of a neuron and its fluorescence levels in false color. BDNF application is indicated by the arrow. (b) Normalized calcium levels in soma ( $n=10)$ and dendrites $(n=16)$. Scale bar $10 \mu \mathrm{m}$. 


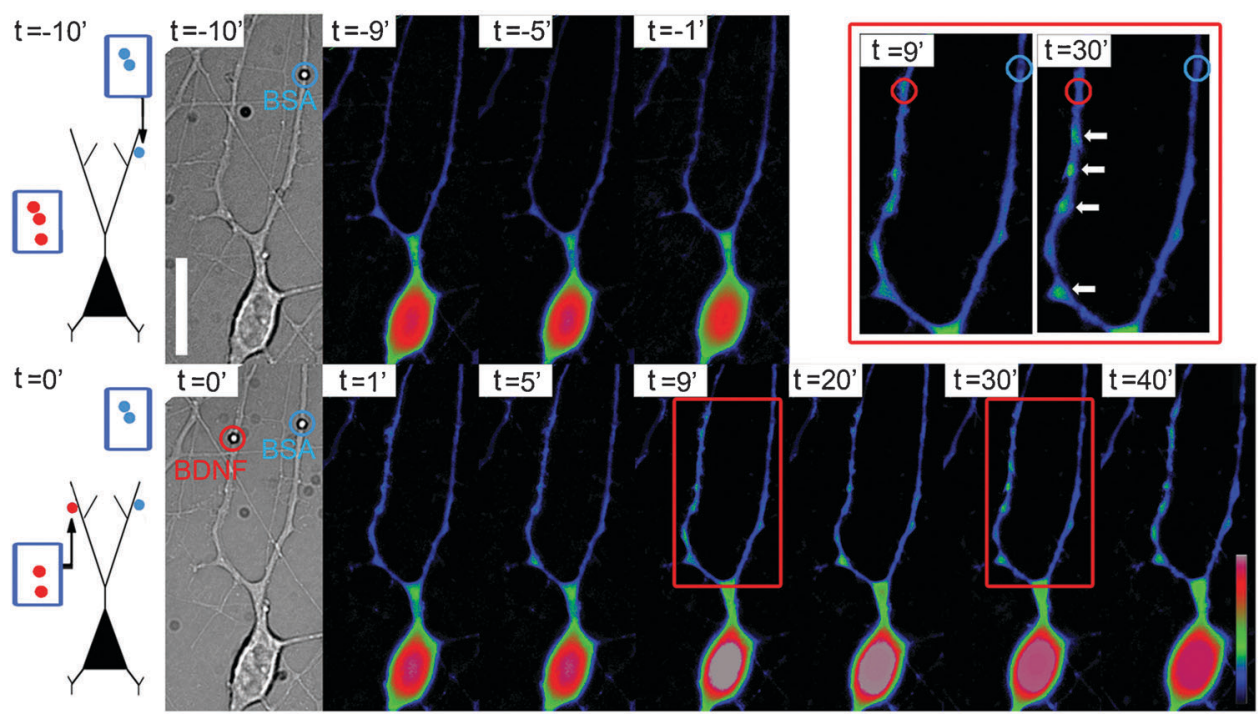

Fig. 5 Focal stimulation of primary rat hippocampal neurons by optically manipulated functionalized beads. Activation of BDNF-dependent calcium signaling pathway. A BSA-bead was placed at $35.5 \mu \mathrm{m}$ from soma (first transmission image), as schematically depicted on the left $\left(t=-10^{\prime}\right)$. Raw calcium levels detected by Fluo-3 fluorescence are visualized in false colors at the indicated times (selection from the image sequence acquired every $2 \mathrm{~min})$. A BDNF-bead was then placed at the same distance from the cell body $\left(t=0^{\prime}\right)$. The fluorescence images show a clear increase in calcium levels within the cell body. In the inset, enlargement of dendrites 9 and $30 \mathrm{~min}$ after stimulation clearly showing a difference between the two stimulated dendrites. Arrows indicate high calcium concentration. Scale bar $15 \mu \mathrm{m}$.

showing an almost constant calcium level, and hence nonactivation of the TrkB receptor for a pair of BSA-beads. After positioning the first BSA-bead (BSA-1), the curves of the control and experimental groups of cells were overlapping (Fig. 6a). When the second bead was coated with BSA (BSA-2) there was no increase in calcium levels in the soma, but when the second bead was coated with BDNF, the curves began to split showing a sudden increase in somatic calcium levels (Fig. 6a). A binning of values between -10 to 0,1 to 10 , 15-25 and 30-40 min outlines this difference, having a significant $p$-value $(<0.001)$ for the last two sets of times (Fig. 6b). The effect on calcium levels obtained with local application of BDNF, was similar to that observed after bath application of $50 \mathrm{ng} \mathrm{mL}^{-1}$ BDNF (Fig. 4b). These results suggest that there was a strong activation of neurons induced by BDNF-functionalized beads, further confirming that BDNF immobilized on the bead surface was active and able to stimulate an intracellular signaling cascade.

To determine if this method allows for local stimulation of TrkB-signaling in the cell periphery, we carried out a semiquantitative analysis of calcium levels in dendrites. BSA-beads did not alter calcium concentration and the curves of the two dendrites in control experiments were superimposed at all times, demonstrating that there was no activation due to the mechanical stimulation of neurons or effects produced by BSA itself (Fig. 6c and d). On the contrary, dendrites stimulated with BDNF-beads showed increased calcium levels and, at 15 and $25 \mathrm{~min}$ after placement of the second bead, a statistically significant difference with respect to dendrites in contact with the BSA-bead, (Fig. 6e and f; $p=0.002$ ). These results indicate selective TrkB activation only in dendrites in contact with BDNF-beads. In dendrites, calcium signals showed a different kinetic behavior compared to soma, starting about 10 min after the stimulation with the neurotrophin-coated bead, but like in the soma, they were prolonged in time. Interestingly, in the last $10 \mathrm{~min}$ of the experiment a rise in calcium concentration, probably caused by ion diffusion, was observed also in the first dendrite stimulated with BSA. This was not surprising since the whole cell (and thus also the control dendrite) was activated, as indicated by the large increase of $\mathrm{Ca}^{++}$in the soma.

\section{Discussion}

Local stimulation has always been an objective in neuroscience, since neurons are morphologically and functionally complex cells and the cellular response to a particular stimulus, critically depends on how and where it is delivered. ${ }^{30}$ In the 1970s, Campenot noticed a specific local effect of the neurotrophin NGF on distal neuritis, causing their growth, while local NGF was not required at the somas and proximal portions of the neurites. ${ }^{31}$ Later, a different response of basal and apical dendrites to the same bath-applied neurotrophin was demonstrated. ${ }^{32}$ More recently, the effect induced by different kinetics in neurotrophin administration was explored $^{33,34}$ and differences in the activation of signaling pathways were found. Techniques controlling both aspects are developing, however they have drawbacks. In fact, most delivery techniques generate gradients of molecules that can diffuse to the nearby region of the cell or have minimal flexibility, since they are efficient only for small compounds (photo-uncaging ${ }^{1}$ ) or require protein engineering (photoactivatable proteins ${ }^{3}$ ).

The use of functionalized beads for local delivery of neurotrophins was proposed by Gallo, ${ }^{11}$ who used $10 \mu \mathrm{m}$ large vectors settled in a cell culture dish or, in later works, microbeads were positioned by means of micropipettes. ${ }^{12,14}$ Considering an average number of 7 spines every $10 \mu \mathrm{m}$ in a 

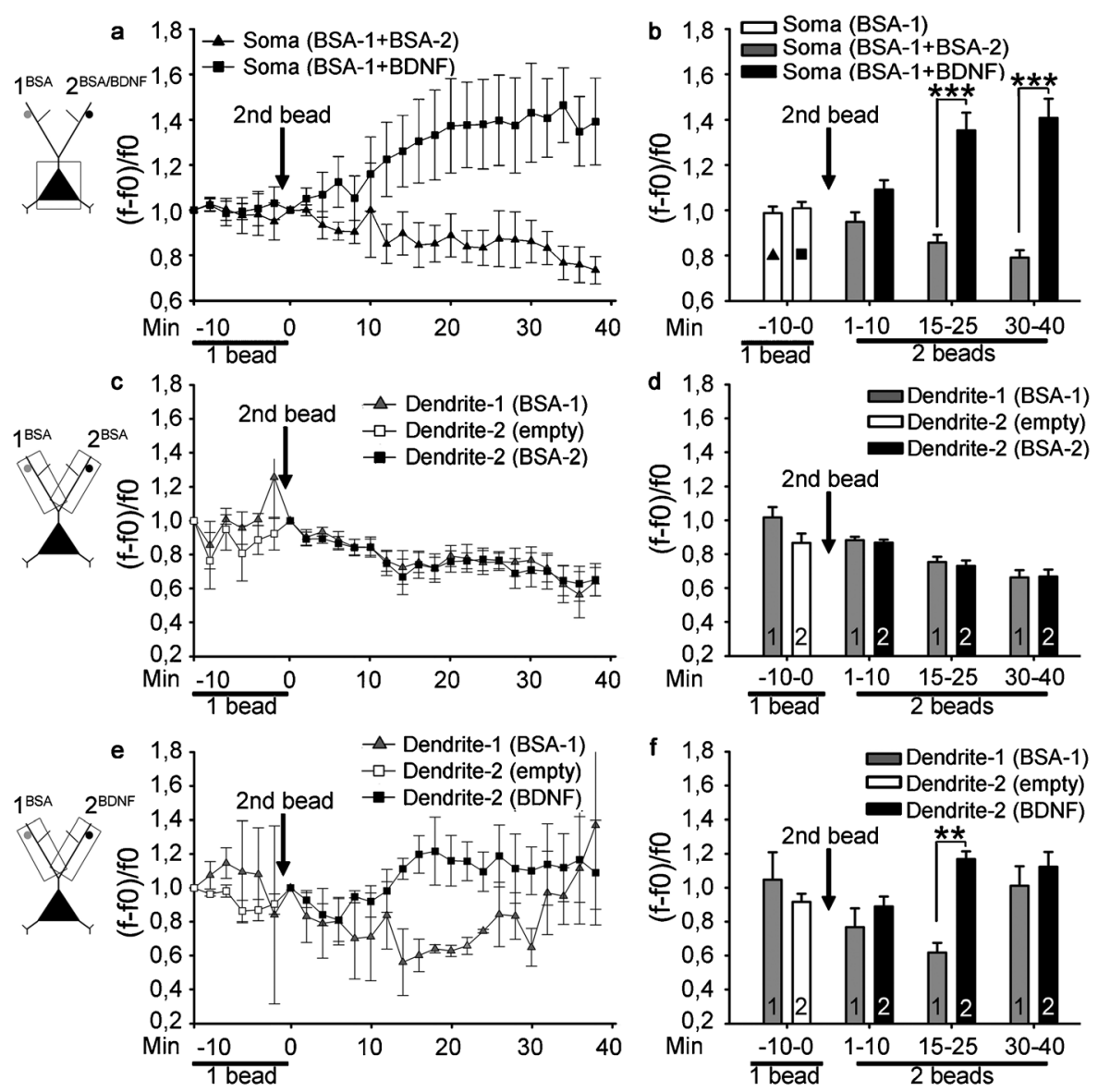

Fig. 6 Neurons stimulation with BDNF-beads increase $\mathrm{Ca}^{++}$levels while BSA-beads do not. (a) Normalized values of calcium levels measured in the cell body of the neurons stimulated with two subsequent BSA-beads (BSA-1 + BSA-2) $(n=5)$ or with a BSA- and then a BDNF-bead $($ BSA-1 + BDNF) $(n=4)$. Arrows indicate the moment of second bead placement. A clear divergence is observed immediately after placing the second bead. (b) Binning of values represented in (a) at the indicated time gaps underlines the differences observed. (c) Normalized values of calcium levels measured along the two dendrites ( $10 \mu \mathrm{m}$ before and after each bead) both stimulated with BSA beads and (d) binning of data. The two curves are almost identical for all the times. (e, f) Same as (c, d) but for dendrites stimulated with BSA and then BDNF. Calcium levels increased in dendrite- 2 about $10 \mathrm{~min}$ after stimulation and values are statistically different between 15 and 25 min. $p$-value: $* *<0.005 ; * * *<0.001$. Analysis performed with one-way ANOVA.

mature hippocampal neuron ${ }^{35}$ it can be immediately understood that this technique does not allow for precise focal stimulation. Smaller magnetic beads $(250 \mathrm{~nm}$ large) were also used, ${ }^{13}$ however these particles are at the limit of optical resolution and their position cannot be controlled with precision. Here we proposed a new approach that, by coupling the use of micro-beads and optical tweezers, allows a high resolution stimulation of cells.

We show that this novel approach has several advantages. First, we demonstrated the possibility of individually manipulating different beads stored in small reservoirs by means of optical tweezers and that several reservoirs with differently coated beads can be used. Secondly, with our system, more than two kinds of beads can be manipulated, analyzing the response of the same cell to each of them. We showed that it is possible to stimulate the same cell simultaneously with one activator (BDNF) and a control (BSA) bead placed on two different dendrites and obtain a specific response on only one dendrite. Thirdly, the use of optical tweezers instead of micropipettes for the manipulation of the vectors has the advantage that the effective contact between the bead and the cell can be easily verified since the particle does not follow the optical trap when it is in contact with the cellular membrane. Fourthly, beads down to $0.3 \mu \mathrm{m}$ diameter can be used and placed on the cell with sub-micrometric precision ensuring focal stimulation of a single spine/ synapse. Moreover, because beads did not move during the 50 min of time-lapse recording, this study demonstrated that this technology is particularly well suited for monitoring longterm responses induced by BDNF. Thus it is possible to study cellular phenomena linked to long term potentiation or depression, such as rearrangements of endoplasmic reticulus, trans-Golgi network and secretory vescicles, mRNA granule trafficking and translation within the spines following BDNF stimulation. ${ }^{36-38}$ These modifications rely on microtubulebased traffic ${ }^{39}$ and need a persistent stimulation to occur, like the one provided by the functionalized beads.

In our study, BDNF was coupled to silica beads through a reaction between the carboxylic groups of the bead and the amine groups of the protein. We demonstrated that BDNF 
was still biologically active because a single functionalized bead in touch with a neuron was able to induce TrkB phosphorylation and a robust c-Fos translocation within the nucleus. Similarly, a single BDNF-coated bead was able both to influence the mobility of growth cones and to induce a large $\mathrm{Ca}^{++}$response in the stimulated dendrites and the soma that, after $30 \mathrm{~min}$, spread also into the unstimulated dendrites. This striking cell-wide activation of TrkB receptor signaling by a single BDNF-bead positioned on a dendrite, represents a particularly important finding of this study because it supports the use of this technology to study cell changes induced by activation of a small set of synapses/spines - if not a single one. Of note, the use of functionalized beads, unlike systems that deliver free molecules, does not allow ligands to be endocytosed. In this way signaling deriving from retrograde transport of the signaling endosomes is not activated. Indeed, distinct biological responses to neurotrophins, and in particular to NGF (i.e. neuronal differentiation or survival), can be controlled by receptors signaling from different locations within the cell. ${ }^{40,41}$ In our study, the possibility that the signal can reach the cell body unaccompanied by the molecule that initiated it, cannot be ruled out. ${ }^{26}$

A local calcium transient caused by local stimulation with BDNF has been reported in the literature, ${ }^{10}$ and has been demonstrated to be independent from internal stores. This transient is fast and can be observed only in the first seconds after stimulation. Since in our setup we need to switch from the camera used for trapping to another camera used for fluorescence imaging, we could not investigate these events. We have a time lag of $30 \mathrm{~s}$ between the actual bead deposition and the first frame of live calcium imaging. Amaral and Pozzo-Miller ${ }^{42,43}$ showed that this signal is able to reach the soma and it induces the activation of the calcium-dependent cationic current $I_{\mathrm{BDNF}}$ mediated by TRPC 3 channels. This causes a further increase of $\mathrm{Ca}^{++}$in dendrites in an internal stores dependent manner.

We can speculate a mechanism similar to that described by Amaral and Pozzo-Miller for the effect we observe, since the timing is comparable. To explore this hypothesis, our setup needs a further modification to allow simultaneous optical manipulation of the microbeads and fluorescence imaging of neurons. The reported experiments seem to prove that no mechanical stimulation of neurons is caused by the beads. However we cannot exclude that a fast, immediate signal was induced by the beads (both BSA and BDNF coated).

In conclusion, here we propose the use of optical tweezers as an approach to stimulate defined neuronal compartments by manipulating functionalized beads. This technique could be useful for studying any growth factor, hormone or peptide signaling at sub-cellular levels and to overcome problems related to other local delivery methods, such as unwanted diffusion of soluble compounds. It has a high flexibility since beads of any dimension can be functionalized with any desired protein or molecule. Its high resolution could allow studies of neural processes at single dendrite level, having the desired controls within the same neuron and thus reducing the variability of the system. The proposed optical manipulation is a simpleto-implement setup, which can easily be applied in biolaboratories not specialized in optical tweezers. The technique might be of interest not only for neurobiology, but also for molecular oncology, immunology and any other field in which single cell response to a specific and localized stimulus is studied.

\section{Acknowledgements}

This work was partially funded by EU FP-7 Projects Nanoscale 214566 and NFFA-Nanoscale Foundries and Fine Analysis 212348 .

\section{References}

1 G. C. Ellis-Davies, Nat. Methods, 2007, 4, 619-628.

2 G. H. Patterson and J. Lippincott-Schwartz, Science, 2002, 297, 1873-1877.

3 Y. I. Wu, D. Frey, O. I. Lungu, A. Jaehrig, I. Schlichting, B. Kuhlman and K. M. Hahn, Nature, 2009, 461, 104-108.

4 G. Nagappan and B. Lu, Trends Neurosci., 2005, 28, 464-471.

5 M. V. Chao, Nat. Rev. Neurosci., 2003, 4, 299-309.

6 L. F. Reichardt, Philos. Trans. R. Soc. London, Ser. B, 2006, 361, $1545-1564$.

7 H. M. Heerssen and R. A. Segal, Trends Neurosci., 2002, 25, $160-165$.

8 A. H. Kossel, S. B. Cambridge, U. Wagner and T. Bonhoeffer, Proc. Natl. Acad. Sci. U. S. A., 2001, 98, 14702-14707.

9 H. W. Horch and L. C. Katz, Nat. Neurosci., 2002, 5, $1177-1184$.

10 S. B. Lang, V. Stein, T. Bonhoeffer and C. Lohmann, J. Neurosci., 2007, 27, 1097-1105.

11 G. Gallo, F. B. Lefcort and P. C. Letourneau, J. Neurosci., 1997, 17, 5445-5454.

12 A. Yoshii and M. Constantine-Paton, Nat. Neurosci., 2007, 10, $702-711$.

13 Y. Naka, A. Kitazawa, Y. Akaishi and N. Shimizu, J. Biosci. Bioeng., 2004, 98, 348-352.

14 X. Zhang and M. M. Poo, Neuron, 2002, 36, 675-688.

15 A. Ashkin, in Optical Trapping and Manipulation of Neutral Particles Using Lasers: A Reprint Volume with Commentaries, World Scientific, River Edge, 2007, pp. 125-187.

16 H. Zhang and K. K. Liu, J. R. Soc. Interface, 2008, 5, 671-690.

17 C. T. Lim, M. Dao, S. Suresh, C. H. Sow and K. T. Chew, Acta Mater., 2004, 52, 1837-1845.

18 E. Ferrari, V. Emiliani, D. Cojoc, V. Garbin, M. Zahid, C. Durieux, M. Coppey-Moisan and E. Di Fabrizio, Microelectron. Eng., 2005, 78-79, 575-581.

19 H. Kress, E. H. Stelzer, D. Holzer, F. Buss, G. Griffiths and A. Rohrbach, Proc. Natl. Acad. Sci. U. S. A., 2007, 104, 11633-11638.

20 D. Cojoc, F. Difato, E. Ferrari, R. B. Shahapure, J. Laishram, M. Righi, E. M. Di Fabrizio and V. Torre, PLoS One, 2007, 2, e1072.

21 B. Sun and D. T. Chiu, J. Am. Chem. Soc., 2003, 125, 3702-3703.

22 H. Kress, J. G. Park, C. O. Mejean, J. D. Forster, J. Park, S. S. Walse, Y. Zhang, D. Wu, O. D. Weiner, T. M. Fahmy and E. R. Dufresne, Nat. Methods, 2009, 6, 905-909.

23 K. C. Neuman and S. M. Block, Rev. Sci. Instrum., 2004, 75, $2787-2809$.

24 E. Tongiorgi, M. Righi and A. Cattaneo, J. Neurosci., 1997, 17, 9492-9505.

25 M. B. Rosenberg, E. Hawrot and X. O. Breakefield, J. Neurochem., 1986, 46, 641-648.

26 J. Du, L. Feng, E. Zaitsev, H. S. Je, X. W. Liu and B. Lu, J. Cell Biol., 2003, 163, 385-395.

27 S. Finkbeiner, S. F. Tavazoie, A. Maloratsky, K. M. Jacobs, K. M. Harris and M. E. Greenberg, Neuron, 1997, 19, 1031-1047.

28 M. Sheng, G. McFadden and M. E. Greenberg, Neuron, 1990, 4, $571-582$.

29 K. Shen and C. W. Cowan, Cold Spring Harbor Perspect. Biol., 2009, 2, a001842.

30 L. F. Reichardt and W. C. Mobley, Cell, 2004, 118, 141-143. 
31 R. B. Campenot, Proc. Natl. Acad. Sci. U. S. A., 1977, 74, 4516-4519.

32 A. K. McAllister, D. C. Lo and L. C. Katz, Neuron, 1995, 15, 791-803.

33 B. L. MacInnis and R. B. Campenot, Science, 2002, 295, $1536-1539$.

34 Y. Ji, Y. Lu, F. Yang, W. Shen, T. T. Tang, L. Feng, S. Duan and B. Lu, Nat. Neurosci., 2010, 13, 302-309.

35 N. L. Desmond and W. B. Levy, Neurosci. Lett., 1985, 54, 219-224.

36 E. Tongiorgi, Neurosci. Res., 2008, 61, 335-346.

37 L. E. Ostroff, J. C. Fiala, B. Allwardt and K. M. Harris, Neuron, 2002, 35, 535-545.
38 H. Toresson and S. G. Grant, Eur. J. Neurosci., 2005, 22 , $1793-1798$.

39 J. Shan, T. P. Munro, E. Barbarese, J. H. Carson and R. Smith, J. Neurosci., 2003, 23, 8859-8866.

40 Y. Zhang, D. B. Moheban, B. R. Conway, A. Bhattacharyya and R. A. Segal, J. Neurosci., 2000, 20, 5671-5678.

41 B. Cui, C. Wu, L. Chen, A. Ramirez, E. L. Bearer, W. P. Li, W. C. Mobley and S. Chu, Proc. Natl. Acad. Sci. U. S. A., 2007, 104, 13666-13671.

42 M. D. Amaral and L. Pozzo-Miller, J. Neurophysiol., 2007, 98 2476-2482.

43 M. D. Amaral, C. A. Chapleau and L. Pozzo-Miller, Pharmacol. Ther., 2007, 113, 394-409. 